\title{
Comparison of tactical offensive variables in different playing surfaces in sided games in soccer
}

\author{
Jorge Diaz-Cidoncha Garcia ${ }^{1,2}$, Ignacio Refoyo Román ${ }^{2}$, Julio Calleja-González ${ }^{3}$ and \\ Alexandre Dellal ${ }^{4}$ \\ ${ }^{1}$ Fédération Internationale de Football Association (FIFA) / Education and Technical \\ Development Department. \\ ${ }^{2}$ National Institute of Physical Education (INEF), Polytechnic University of Madrid / \\ Sports Department. \\ ${ }^{3}$ National Institute of Physical Education (INEF), University of Basque Country. \\ ${ }^{4}$ FIFA Medical Centre of Excellence, Centre Orthopédique Santy. Lyon, France.
}

\begin{abstract}
In recent decades soccer has been analysed in great detail, enabling the technical and tactical aspects of the game to be improved through observation, analysis and training. However, there are not significant studies that particularly looked at the impact of the playing surfaces in depth. Thus the main aim of this study is to analyse and compare the quantitative and qualitative effect of the playing surface (grass, dirt or turf) on teams' and players' performances from a tactical perspective, through observing various sided games in youth soccer players. 54 games in three different formats $(5 v 5,7 v 7$ and $9 v 9)$ were analysed, divided into two age groups (378 players of $U-9$ and $U-14$ categories) and filmed at three Spanish soccer clubs. This study used the observational and descriptive method and was carried out by systematic observation. This involved watching pre-recorded matches to observe and log different types of behaviour in previously defined categories. Results revealed that there were no significant differences for the different surfaces or game formats, and the study presents a description and analysis of the aspects that had considerable influence on the attacking moves for each surface and game format.
\end{abstract}

Key words: soccer, attacking, sided game.

\section{Introduction}

1.1. Playing surfaces in soccer

Different types of playing surfaces are available for soccer, the choice depending on the characteristics and requirements of each sports facility. Until a few years ago, the most common surfaces were dirt and natural grass, although artificial turf has grown in popularity in the last decade (Duncan et al., 2002). Natural grass continues to be the most widely used option for soccer pitches, although their use may be limited by 
weather environmental conditions and because of the problem of deterioration (especially in grassroots soccer when pitches often are used for many hours a day), which presents that the use of the pitches for training may be restricted because of the need to keep them in optimum condition for competitions. More specifically, the type of grass will determine the capacity of the natural surfaces to drain adequately and the degree of compaction of the earth (Lees and Nolan, 1998). There are other properties of natural surfaces that may have an impact on the development of play, such as: unevenness, hardness and friction (Ekstrand, 2008). It should be noted that many soccer grounds at grassroots level do not receive the maintenance necessary to keep the pitches in good condition (FIFA, 2010). For example, natural grass requires a lot of attention throughout the year: mowing, watering, and preventing and dealing with pests, weeds and diseases (Stiles et al., 2009). Meanwhile, dirt pitches are becoming less common, although there are many soccer grounds with this type of surface, especially in areas with adverse climate conditions and water scarcity. This type of surface does not require much maintenance, but it is not very even and is very hard. It should be pointed out that dirt was the most common type of surface in recent decades in grassroots and youth soccer as well as in amateur soccer.

Third-generation (3G) artificial soccer turf was designed specifically for soccer and attempted to replicate the characteristics of natural grass (Sanchís et al., 2008). SchmidtOltsen et al. (1991) explained that artificial turf was proposed as an alternative to avoid the main drawbacks of natural grass, in particular in areas with unfavourable climatic conditions. However, it had high maintenance costs, and both rain and ice increased the risk of injuries due to deterioration of parts of the pitch and reduction of the surface's flexibility. It must also be noted that, despite the technology having become more advanced, artificial turf was somewhat rigid, retained a lot of heat and was slipperier than natural surfaces (Orchard, 2002). Nowadays, fourth-generation artificial turf has been developed to minimise the drawbacks that the previous versions had in comparison with natural turf (Claudio, 2008). The Fédération Internationale de Football Association (FIFA) has developed the FIFA Quality Programme for Soccer Turf (FIFA, 2013), and this type of surface has been used in various international elite youth competitions since 2005, when the 32 matches of the U-17 World Championship in Peru were played on artificial pitches. Nevertheless, all types of playing surfaces, whether they are natural grass, artificial turf or dirt, may use different materials, all of which will have different characteristics (Steffen et al., 2007).

\subsection{Small-sided soccer}

Over the last decade, small-sided games in soccer have become more common drills, whether in organised or spontaneous set-ups. These types of games offer many possibilities and combinations that increase the level of interaction among the players (Platt et al, 2001; Tessitore et al, 2006). Sided soccer is very beneficial for participants, especially for learning purposes in the youth age categories and for training in senior age categories. Sided soccer means from $4 \mathrm{v} 4$ to $9 \mathrm{v} 9$. On the other hand, the pitch dimensions are smaller than in 11v11 and the rules are adapted to each format (e.g. the goals and the areas are smaller). Previous studies have shown that players have more touches of the ball, progress more quickly, take more decisions during the match, and increased levels of concentration and intensity are called for as the ball is never far away (Rampinini et al, 2007; Hill-Haas et al, 2009; Abrantes et al, 2012). Participation 
levels are also increased as having fewer players on the pitch means participants have to be more attentive (Hill-Haas et al., 2010). In addition, there will be more attacking and defending situations as the players are continually under pressure in small-sided games (Casamichana and Castellano, 2010; FIFA, 2012).

\subsection{Performance analysis - tactical variables}

Performance analysis has become a widespread practice across a range of sports and disciplines, including soccer. Despite becoming increasingly common, it has mainly been restricted to professional or elite levels of sport, although in the last few years performance analysis techniques have started to be used in soccer academies as well. At grassroots level, as the necessary technology has become cheaper, implementing performance analysis processes has become more accessible. For example, a grassroots coach with a laptop and a home video camera can now analyse the performance of the tactical variables of the players. Performance analysis research is generally applied research, though there has been some basic theoretical research. The levels of development and sophistication of the methods employed have increased, enabling more extensive data to be analysed more accurately. Some of the topics mentioned by O'Donoghue (2005) that are relevant for this study concluded: critical incidents and occurrences (Atkinson, 2002), performance indicators in various disciplines (O'Donoghue et al, 2007), reliability of methods (Choi et al, 2007), tactical patterns of play (Reilly et al, 2000), performance sampling (Hadley et al, 2000), and effectiveness of the support tools for the performance analysis (James et al, 2007). With these indicators it is possible to determine the action variables that define some or all aspects of performance and often have an effect on the outcome of moves (Hughes \& Bartlett, 2002). Thus, the aim of this study is to analyse and compare quantitative and qualitative impacts of the type of surface and game format on the performance of the team and the players, in order to determine the effect of these elements upon specific tactical results.

\section{Methods}

\subsection{Participants}

A sample of 54 grassroots soccer games played by boys, in the U-9 and U-14 age groups was analysed to collect the data for this study (for U-9: height $134.1 \pm 12.3 \mathrm{~cm}$; body mass $29.4 \pm 11.6 \mathrm{~kg}$ and for U-14: height $163.0 \pm 13.8 \mathrm{~cm}$; body mass $52.9 \pm 13.1$ $\mathrm{kg}$ ). The U-9 group had an experience of 3 years playing organised soccer, while the U14 group had an experience of 6 years. The same teams were monitored for a specific period of time to ensure that the sample was as broad as possible. The games for the sample were also selected, taking into account game formats $(5 v 5,7 v 7$ and 9v9), types of playing surface (natural grass, artificial turf, dirt), as well as weather conditions. The games were recorded at three different clubs in Spain: the soccer academy of the Spanish Soccer Association (RFEF), Adarve-Barrio del Pilar and Villanueva del Pardillo. Of the 54 games in the sample, the following recordings (and subsequent analyses) were made: $36 \times 2$ for each age group (U-9 and U-14); $18 \times 3$ on each playing surface (natural grass, artificial turf, dirt); $18 \times 3$ in each game format $(5 v 5,7 v 7,9 v 9)-$ having recorded 3 matches for each cell of the design. Each game lasted 20 minutes, with no breaks and no substitutions. 


\subsection{Measures}

The stages used in the observational method are as follows: formulating a problem, collecting and recording the data, analysing and interpreting the observed data and communicating the results. Moreover, an initial assessment was carried out before each game to ensure that the conditions relating to the size and quality of the pitch, weather, support facilities for the AV recording, etc. were acceptable. At the end of each game, each player answered a series of questions assessing their enjoyment of the game, their impressions of the pitch, their perceptions of their own level of participation and the level of external pressure they felt they were under. The study aimed to reflect a range of contexts and real-life situations, because there is not just one type of sporting practice, but several, and they take place in a wide variety of contexts (Andersson et al, 2008). Therefore, a highly representative sample covering all of the possible parameters that may influence the development of the game was selected.

\subsection{Procedures}

Fifty- four games were used for the study, all of which were recorded on video. All games were watched live and were then analysed with the aid of the video recordings, with the same process being used for each and the analysis being performed by at least three different reviewers. Data was collected from watching the recordings, monitoring predefined variables. The study is descriptive, carried out using a systematic and quantitative observational method that was prepared in advance and took place in a reallife setting. The observational method is a particular type of scientific methodology that may be used with the aim of quantifying spontaneous behaviour occurring in natural situations, and it must be carried out following a specific series of stages (O'Donoghue, 2009). The collection of the data and its analysis were approved by the relevant ethics regulatory body and was also performed in compliance with the ethical principles for research involving human subjects as specified in the Helsinki Declaration (2008). This study also complies with the laws of data protection, as well as with the ethics committee of the Polytechnic University of Madrid's Institutional Review Board.

\subsection{Equipment - instruments}

The equipment used in the study consisted of balls (size 4 for U-9 and size 5 for U-14), different coloured bibs, cones and scoreboards, mini-goals and seven-a-side goals (depending on the game format). The games were timed by using a Traceable digital stopwatch. The games were recorded by using a Sony HDR-CX570 camera and a HIPOD tripod. When all of the planned sessions had been recorded, the games were watched on a TV monitor and analysed.

\subsection{Statistical analysis}

After applying a Shapiro-Wilk normality test (revealing a normal distribution of the data), the arithmetic mean and standard deviation of each variable observed in the footage of the 54 games were calculated. Parametric tests were applied, using SPSS 22.0 pack (Illinois, Chicago, USA) for every statistic calculation. No relevant interactions were identified after applying a three way Anova with significance accepted at the $\mathrm{p} \leq 0.05$ level, and Cohen's Kappa has been analysed for the most frequent performance indicators showing that the results for the representative variables presented a substantial degree of concordance of 0.61-0.80. For the percentage indicators (time and pitch zones), the percentages for each of them were also compared. 


\section{Results}

Analysing the most relevant performance indicators, the proportion of attacks launched from a pass forward was greater on artificial turf $(55 \%)$ than on dirt $(50 \%)$ or natural grass (44\%); a greater number of attacks on artificial pitches ended in a shot (57\%), in comparison with $52 \%$ on dirt pitches and $51 \%$ on natural grass. Regarding goals scored, a greater number were scored on artificial turf $(22 \%)$ than on dirt $(17 \%)$ or natural grass $(15 \%)$. Meanwhile, the proportion of counter-attacks was greater on artificial turf $(71 \%)$ than on dirt $(68 \%)$ or natural grass $(58 \%)$. The average number of attacks was similar on all the surfaces, although slightly more attacks were observed on artificial turf (14) and natural grass (14) than on dirt pitches (12). The proportion of counter-attacks was significantly higher on artificial turf $(71 \%)$ and dirt pitches $(68 \%)$ than on natural grass $(58 \%)$. The significant majority of all attacks were initiated by a pass forward, although the proportion of attacks started in this way was higher on artificial turf (55\%) than on dirt pitches $(50 \%)$ or on natural grass $(43 \%)$. In the majority of cases, the attack ended after a challenge by the opposition's goalkeeper or defenders $(59 \%$ on dirt, $54 \%$ on natural grass and $48 \%$ on artificial turf). However, it was notable that in the $5 \mathrm{v} 5$ game format on natural grass, the proportion of attacks ended by an attacker or ending in a goal $(46 \%)$ was significantly higher than the overall average $(33 \%)$. On all surfaces, there was a fairly equal split between attacks ending with either a loss of possession or a shot. Nevertheless, attacks ended in a shot more often on artificial turf pitches $(59 \%)$ than on dirt pitches $(54 \%)$ or natural grass $(57 \%)$. On the other hand, those attacks led to a greater number of goals on artificial turf $(22 \%)$ than on dirt $(17 \%)$ or natural grass $(15 \%)$. It was also notable that on dirt pitches, the 5v5 game format generated more shots on target $(47.5 \%)$ than the overall average $(33 \%)$ and proportionally fewer incidences of loss of possession (25.5\%) than the average (46\%). 
Table 1. Summary of the tactical variables for soil.

\begin{tabular}{|c|c|c|c|c|c|c|c|c|c|c|c|c|c|c|c|c|c|}
\hline \multirow{2}{*}{ Variables } & \multicolumn{2}{|c|}{ U-9 5 5v5 } & \multicolumn{2}{|c|}{ U-9 7v7 } & \multicolumn{2}{|c|}{ U-9 9v9 } & \multicolumn{2}{|c|}{ U-14 5v5 } & \multicolumn{2}{|c|}{ U-14 7v7 } & \multicolumn{2}{|c|}{ U-14 9v9 } & \multirow{2}{*}{ MEAN } & \multirow{2}{*}{$\%$} & \multirow{2}{*}{ SD } & \multirow{2}{*}{ Range } & \multirow{2}{*}{ CI } \\
\hline & Avg & $\%$ & Avg & $\%$ & Avg & $\%$ & Avg & $\%$ & Avg & $\%$ & Avg & $\%$ & & & & & \\
\hline No. of attacks & 19 & & 10 & & 11 & & 10 & & 12 & & 9 & & 12 & - & 4,61 & 21 & $9,71-14,29$ \\
\hline \multicolumn{18}{|l|}{ Origin of attack } \\
\hline Defensive half & 14 & 72 & 6 & 62 & 4 & 35 & 8 & 74 & 7 & 57 & 5 & 38 & 7 & $60 \%$ & 4,09 & 17 & 5,13-9,20 \\
\hline Attacking half & 5 & 28 & 4 & 38 & 7 & 65 & 3 & 26 & 5 & 43 & 5 & 38 & 5 & $40 \%$ & 2,64 & 11 & $3,52-6,15$ \\
\hline \multicolumn{18}{|c|}{ How move was initiated } \\
\hline Counter-attack & 14 & 74 & 7 & 69 & 9 & 76 & 7 & 68 & 8 & 62 & 5 & 41 & 8 & $68 \%$ & 3,63 & 12 & $6,36-9,97$ \\
\hline Static attack & 5 & 26 & 3 & 31 & 3 & 24 & 3 & 32 & 5 & 38 & 4 & 35 & 4 & $32 \%$ & 2,46 & 11 & $2,61-5,05$ \\
\hline \multicolumn{18}{|c|}{ Initiating player } \\
\hline GK & 4 & 21 & 0.3 & 3 & 1 & 9 & 3 & 29 & 1 & 8 & 1.7 & 14 & 2 & $15 \%$ & 1,79 & 7 & $0,94-2,72$ \\
\hline Defender & 7 & 37 & 4 & 38 & 1 & 12 & 4 & 39 & 3 & 27 & 3 & 24 & 4 & $31 \%$ & 2,67 & 12 & $2,39-5,05$ \\
\hline Midfielder & 0 & 0 & 4 & 38 & 6 & 53 & 0 & 0 & 4 & 35 & 4 & 32 & 3 & $25 \%$ & 2,47 & 7 & $1,77-4,23$ \\
\hline \begin{tabular}{|l|} 
Attacker \\
\end{tabular} & 8 & 42 & 2 & 21 & 3 & 26 & 3 & 32 & 4 & 30 & 1 & 5 & 3 & $29 \%$ & 2,75 & 11 & $2,08-4,81$ \\
\hline \multicolumn{18}{|c|}{ How the ball was pushed forward } \\
\hline Pass & 9 & 46 & 6 & 66 & 6 & 56 & 4 & 39 & 7 & 59 & 4 & 30 & 6 & $50 \%$ & 2,55 & 10 & $4,79-7,33$ \\
\hline Goal kick & 2 & 11 & 0 & 3 & $\mathbf{0}$ & 0 & 0 & 3 & 0 & 3 & 1 & 5 & 1 & $5 \%$ & 1,20 & 5 & $0,02-1,21$ \\
\hline GK throw & 2 & 9 & $\mathbf{0}$ & 0 & 1 & 6 & 2 & 19 & $\mathbf{0}$ & 3 & 1 & 5 & 1 & $7 \%$ & 0,96 & 3 & $0,41-1,37$ \\
\hline Corner & 1 & 5 & 1 & 10 & 1 & 9 & 1 & 6 & 1 & 11 & 1 & 11 & 1 & $9 \%$ & 1,06 & 3 & $0,53-1,58$ \\
\hline Throw-in & 1 & 4 & 1 & 7 & $\mathbf{0}$ & 3 & 1 & 13 & 1 & 8 & 2 & 14 & 1 & $8 \%$ & 0,87 & 3 & $0,51-1,38$ \\
\hline Free kick & $\mathbf{0}$ & 0 & $\mathbf{0}$ & 0 & $\mathbf{0}$ & 3 & $\mathbf{0}$ & 0 & $\mathbf{0}$ & 3 & $\mathbf{0}$ & 3 & $\mathbf{0}$ & $1 \%$ & 0,38 & 1 & $-0,02-0,36$ \\
\hline Dribble & 4 & 19 & 1 & 14 & 1 & 12 & 2 & 19 & 1 & 11 & 0 & 3 & 2 & $14 \%$ & 1,57 & 6 & $0,89-2,45$ \\
\hline Shot & 1 & 5 & 0 & 0 & 1 & 9 & 0 & 0 & 0 & 0 & 0 & 0 & 0 & $3 \%$ & 0,59 & 2 & $0,04-0,63$ \\
\hline Header & $\mathbf{0}$ & 0 & 0 & 0 & 0 & 3 & 0 & 0 & 0 & 3 & 0 & 0 & 0 & $1 \%$ & 0,32 & 1 & $-0,05-0,27$ \\
\hline \begin{tabular}{|l|} 
Penalty \\
\end{tabular} & 0.3 & 2 & $\mathbf{0}$ & 0 & $\mathbf{0}$ & 0 & $\mathbf{0}$ & 0 & $\mathbf{0}$ & 0 & 0.6 & 5 & $\mathbf{0}$ & $1 \%$ & 0,38 & 1 & $-0,02-0,36$ \\
\hline \multicolumn{18}{|c|}{ Area where first pass was received } \\
\hline \begin{tabular}{|l|} 
Defensive half \\
\end{tabular} & 6 & 30 & 4 & 38 & 1 & 9 & 4 & 42 & 5 & 41 & 3 & 24 & 4 & $31 \%$ & 1,90 & 7 & $2,83-4,72$ \\
\hline \begin{tabular}{|l} 
Attacking half \\
\end{tabular} & 11 & 56 & 5 & 52 & 9 & 79 & 5 & 45 & 6 & 51 & 5 & 43 & 7 & $57 \%$ & 3,03 & 13 & $5,32-8,34$ \\
\hline N/A & 3 & 14 & 1 & 10 & 1 & 12 & 1 & 13 & 1 & 8 & 1 & 8 & 1 & $12 \%$ & 1,33 & 5 & $0,73-2,05$ \\
\hline \multicolumn{18}{|c|}{ No. of passes in attack } \\
\hline \begin{tabular}{|l|} 
Average \\
\end{tabular} & 2 & - & 3 & & 2 & - & 3 & - & 3 & & 3 & - & 3 & - & 0,51 & 1 & $2,24-2,76$ \\
\hline \multicolumn{18}{|c|}{ No. of players involved in attack } \\
\hline \begin{tabular}{|l} 
Average \\
\end{tabular} & 3 & & 3 & & 3 & & 3 & & 3 & & 3 & & 3 & - & 0,54 & 2 & $2,79-3,32$ \\
\hline \multicolumn{18}{|c|}{ Duration of attack } \\
\hline Average & 12 & - & 12 & & 11 & & 12 & & 11 & & 12 & & 12 & - & 1,78 & 7 & $11,00-12,77$ \\
\hline
\end{tabular}


Player who ended the attack

\begin{tabular}{|c|c|c|c|c|c|c|c|c|c|c|c|c|c|c|c|c|c|}
\hline Opposition GK & 6 & 33 & 3 & 28 & 3 & 26 & 2 & 19 & 3 & 22 & 4 & 30 & 3 & $28 \%$ & 2,25 & 8 & $2,27-4,51$ \\
\hline Opposition def. & 5 & 25 & 3 & 31 & 5 & 41 & 2 & 19 & 5 & 38 & 3 & 27 & 4 & $31 \%$ & 1,99 & 8 & $2,73-4,71$ \\
\hline Opposition mid. & 0 & 0 & 1 & 14 & 1 & 6 & $\mathbf{0}$ & 0 & 1 & 8 & 0 & 3 & 1 & $5 \%$ & 0,78 & 2 & $0,17-0,95$ \\
\hline Opposition att. & $\mathbf{0}$ & 0 & 0 & 0 & $\mathbf{0}$ & 0 & $\mathbf{0}$ & 0 & $\mathbf{0}$ & 0 & $\mathbf{0}$ & 0 & 0 & $0 \%$ & 0,00 & $\mathbf{0}$ & - \\
\hline Defender & 1 & 7 & 0 & 0 & $\mathbf{0}$ & 3 & 2 & 16 & $\mathbf{0}$ & 0 & $\mathbf{0}$ & 3 & 1 & $5 \%$ & 0,98 & 3 & $0,12-1,10$ \\
\hline Midfielder & $\mathbf{0}$ & 0 & 1 & 10 & $\mathbf{0}$ & 3 & $\mathbf{0}$ & 0 & $\mathbf{0}$ & 0 & $\mathbf{0}$ & 3 & 0 & $2 \%$ & 0,57 & 2 & $-0,01-0,56$ \\
\hline Attacker & 2 & 12 & 1 & 14 & 1 & 9 & 1 & 13 & 2 & 19 & 0 & 3 & 1 & $12 \%$ & 1,15 & 3 & $0,87-2,02$ \\
\hline N/A (Goal) & 4 & 23 & 0 & 3 & 1 & 12 & 3 & 32 & 2 & 14 & 1 & 8 & 2 & $17 \%$ & 1,71 & 6 & $1,15-2,85$ \\
\hline \multicolumn{18}{|l|}{ Result } \\
\hline Shot on target & 8 & 40 & 1 & 14 & 3 & 26 & 6 & 55 & 4 & 30 & 3 & 22 & 4 & $33 \%$ & 2,77 & 11 & $2,62-5,38$ \\
\hline Shot off target & 4 & 19 & 2 & 21 & 2 & 21 & 3 & 26 & 1 & 5 & 2 & 16 & 2 & $19 \%$ & 1,73 & 6 & $1,36-3,08$ \\
\hline Shot blocked & 1 & 5 & 0 & 0 & $\mathbf{0}$ & 0 & 0 & 3 & $\mathbf{0}$ & 3 & $\mathbf{0}$ & 0 & 0 & $2 \%$ & 0,57 & 2 & $-0,01-0,56$ \\
\hline Loss of poss. & 7 & 35 & 6 & 66 & 6 & 53 & 2 & 16 & 8 & 62 & 5 & 38 & 6 & $46 \%$ & 2,64 & 8 & $4,19-6,81$ \\
\hline
\end{tabular}

$\mathrm{SD}=$ standard deviation; $\mathrm{CI}=$ confidence interval; $\mathrm{GK}=$ goalkeeper; def. = defender; mid. = midfielder; att. = attacker; N/A = not applicable; poss. $=$ possession 
Table 2. Summary of the tactical variables for grass.

\begin{tabular}{|c|c|c|c|c|c|c|c|c|c|c|c|c|c|c|c|c|c|}
\hline \multirow{2}{*}{ Variables } & \multicolumn{2}{|c|}{ U-9 5v5 } & \multicolumn{2}{|c|}{ U-9 7v7 } & \multicolumn{2}{|c|}{ U-9 9v9 } & \multicolumn{2}{|c|}{ U-14 5v5 } & \multicolumn{2}{|c|}{ U-14 7v7 } & \multicolumn{2}{|c|}{ U-14 9v9 } & \multirow{2}{*}{ MEAN } & \multirow{2}{*}{$\%$} & \multirow{2}{*}{ SD } & \multirow{2}{*}{ Range } & \multirow{2}{*}{ CI } \\
\hline & Avg & $\%$ & Avg & $\%$ & Avg & $\%$ & Avg & $\%$ & Avg & $\%$ & Avg & $\%$ & & & & & \\
\hline No. of attacks & 19 & & 15 & & 9 & & 12 & & 13 & & 16 & & 14 & - & 4,70 & 19 & $11,61-16,28$ \\
\hline \multicolumn{18}{|l|}{ Origin of attack } \\
\hline Defensive half & 13 & 70 & 7 & 48 & 4 & 50 & 8 & 68 & 7 & 51 & 7 & 56 & 8 & $56 \%$ & 3,17 & 12 & $6,20-9,36$ \\
\hline Attacking half & 6 & 30 & 8 & 52 & 4 & 50 & 4 & 32 & 6 & 49 & 9 & 69 & 6 & $44 \%$ & 3,35 & 14 & $4,50-7,83$ \\
\hline \multicolumn{18}{|c|}{ How move was initiated } \\
\hline Counter-attack & 13 & 70 & 7 & 50 & 6 & 65 & 6 & 46 & 6 & 49 & 10 & 79 & 8 & $58 \%$ & 3,46 & 12 & $6,64-9,77$ \\
\hline Static attack & 6 & 30 & 7 & 50 & 3 & 35 & 7 & 54 & 7 & 51 & 6 & 46 & 6 & $42 \%$ & 2,70 & 11 & $4,55-7,23$ \\
\hline \multicolumn{18}{|c|}{ Initiating player } \\
\hline GK & 4 & 21 & 3 & 20 & $\mathbf{0}$ & 0 & 3 & 24 & 2.3 & 18 & 3.7 & 28 & 3 & $19 \%$ & 2,09 & 7 & $1,63-3,70$ \\
\hline \begin{tabular}{|l|} 
Defender \\
\end{tabular} & 6 & 34 & 5 & 36 & 3 & 38 & 5 & 38 & 3 & 21 & 3 & 21 & 4 & $30 \%$ & 1,95 & 7 & $3,20-5,14$ \\
\hline Midfielder & 0 & 0 & 4 & 27 & 5 & 54 & 0 & 0 & 6 & 44 & 7 & 56 & 4 & $26 \%$ & 3,47 & 12 & $1,89-5,33$ \\
\hline Attacker & 8 & 45 & 4 & 24 & 1 & 12 & 5 & 38 & 2 & 18 & 3 & 21 & 4 & $25 \%$ & 3,15 & 11 & $\begin{array}{l}2,37-5,50 \\
\end{array}$ \\
\hline \multicolumn{18}{|c|}{ How the ball was pushed forward } \\
\hline Pass & 7 & 38 & 6 & 39 & 4 & 50 & 6 & 49 & 5 & 36 & 8 & 64 & 6 & $43 \%$ & 2,50 & 12 & $4,76-7,24$ \\
\hline Goal kick & 2 & 9 & 0 & 2 & 0 & 0 & 1 & 11 & 1 & 10 & 0 & 3 & 1 & $6 \%$ & 0,99 & 3 & $0,34-1,32$ \\
\hline GK throw & 2 & 9 & 0 & 2 & $\mathbf{0}$ & $\overline{0}$ & 1 & 5 & $\mathbf{0}$ & 3 & $\mathbf{0}$ & 0 & 1 & $4 \%$ & 0,71 & 2 & $0,15-0,85$ \\
\hline Corner & 2 & 9 & 2 & 16 & 1 & 12 & 2 & 14 & 2 & 18 & 3 & 26 & 2 & $15 \%$ & 1,66 & 5 & $1,23-2,88$ \\
\hline Throw-in & 1 & 5 & 3 & 23 & 2 & 19 & 2 & 14 & 2 & 18 & 2 & 13 & 2 & $14 \%$ & 1,39 & 5 & $1,25-2,64$ \\
\hline Free kick & $\mathbf{0}$ & 2 & $\mathbf{0}$ & 0 & $\mathbf{0}$ & 4 & $\mathbf{0}$ & 3 & $\mathbf{0}$ & 0 & $\mathbf{0}$ & 0 & $\mathbf{0}$ & $1 \%$ & 0,38 & 1 & $-0,02-0,36$ \\
\hline Dribble & 4 & 20 & 2 & 16 & 1 & 15 & 0 & 3 & 2 & 13 & 2 & 15 & 2 & $14 \%$ & 1,37 & 5 & $1,21-2,57$ \\
\hline \begin{tabular}{|l|} 
Shot \\
\end{tabular} & 2 & 9 & 0 & 2 & 0 & 0 & 0 & 0 & 0 & 3 & 0 & 0 & 0 & $3 \%$ & 0,70 & 2 & $0,04-0,74$ \\
\hline Header & 0 & 0 & 0 & 0 & 0 & 0 & 0 & 3 & 0 & 0 & 1 & 5 & 0 & $1 \%$ & 0,51 & 2 & $-0,09-0,42$ \\
\hline \begin{tabular}{|l|} 
Penalty \\
\end{tabular} & $\mathbf{0}$ & 0 & $\mathbf{0}$ & 0 & $\mathbf{0}$ & 0 & $\mathbf{0}$ & & $\mathbf{0}$ & 0 & $\mathbf{0}$ & 0 & $\mathbf{0}$ & $0 \%$ & 0,00 & $\mathbf{0}$ & - \\
\hline \multicolumn{18}{|c|}{ Area where first pass was received } \\
\hline \begin{tabular}{|l|} 
Defensive half \\
\end{tabular} & 7 & 38 & 3 & 18 & 1 & 12 & 8 & 62 & 5 & 36 & 5 & 36 & 5 & $33 \%$ & 2,93 & 11 & $3,15-6,07$ \\
\hline \begin{tabular}{|l} 
Attacking half \\
\end{tabular} & 8 & 41 & 11 & 77 & 7 & 77 & 3 & 22 & 6 & 46 & 10 & 79 & 7 & $53 \%$ & 3,84 & 14 & $5,54-9,35$ \\
\hline \begin{tabular}{|l|l} 
N/A \\
\end{tabular} & 4 & 21 & 1 & 5 & 1 & 12 & 2 & 16 & 2 & 18 & 1 & 10 & 2 & $14 \%$ & 1,49 & 4 & $1,15-2,63$ \\
\hline \multicolumn{18}{|c|}{ No. of passes in attack } \\
\hline \begin{tabular}{|l|} 
Average \\
\end{tabular} & 2 & - & 2 & - & 2 & - & 3 & - & 3 & - & 3 & - & 3 & - & 0,78 & 3 & $2,17-2,95$ \\
\hline \multicolumn{18}{|c|}{ No. of players involved in attack } \\
\hline \begin{tabular}{|l} 
Average \\
\end{tabular} & 2 & & 3 & & 3 & & 3 & & 3 & & 3 & & 3 & - & 0,64 & 2 & $\begin{array}{l}2,63-3,26 \\
\end{array}$ \\
\hline \multicolumn{18}{|c|}{ Duration of attack } \\
\hline \begin{tabular}{|l|} 
Average \\
\end{tabular} & 10 & - & 8 & - & 10 & - & 13 & - & 14 & - & 12 & - & 11 & - & 2,46 & 8 & $9,95-12,39$ \\
\hline
\end{tabular}




\begin{tabular}{|c|c|c|c|c|c|c|c|c|c|c|c|c|c|c|c|c|c|}
\hline \multicolumn{18}{|c|}{ Player who ended the attack } \\
\hline Opposition GK & 7 & 39 & 4 & 25 & 2 & 23 & 4 & 30 & 4 & 33 & 5 & 36 & $\overline{4}$ & $31 \%$ & 2,37 & 8 & $3,10-5,46$ \\
\hline Opposition def. & 2 & 11 & 5 & 36 & 3 & 31 & 2 & 19 & 2 & 15 & 5 & 38 & 3 & $23 \%$ & 1,99 & 6 & $2,23-4,21$ \\
\hline Opposition mid. & 0 & 0 & 1 & 5 & 1 & 8 & 0 & 0 & 1 & 8 & 2 & 13 & $\overline{1}$ & $5 \%$ & 0,91 & 3 & $0,22-1,12$ \\
\hline Opposition att. & $\mathbf{0}$ & 2 & $\mathbf{0}$ & 2 & $\mathbf{0}$ & 0 & $\overline{0}$ & 0 & $\mathbf{0}$ & 3 & $\mathbf{0}$ & 0 & $\overline{0}$ & $1 \%$ & 0,38 & $\mathbf{1}$ & $-0,02-0,36$ \\
\hline Defender & 1 & 5 & 1 & 5 & $\mathbf{0}$ & 0 & $\mathbf{0}$ & 3 & $\mathbf{0}$ & 3 & $\mathbf{0}$ & 0 & $\overline{0}$ & $3 \%$ & 0,61 & 2 & $0,09-0,69$ \\
\hline Midfielder & $\mathbf{0}$ & 0 & 1 & 5 & 1 & 15 & $\overline{0}$ & 0 & 2 & 18 & 2 & 15 & $\overline{1}$ & $7 \%$ & 1,37 & 4 & $0,32-1,68$ \\
\hline Attacker & 3 & 18 & 3 & 18 & $\mathbf{1}$ & 15 & 3 & 27 & 1 & 8 & 2 & 13 & 2 & $16 \%$ & 1,52 & 6 & $1,47-2,98$ \\
\hline N/A (Goal) & 5 & 25 & 1 & 5 & 1 & 8 & 3 & 22 & 2 & 13 & 2 & 15 & 2 & $15 \%$ & 1,59 & 5 & $1,27-2,85$ \\
\hline \multicolumn{18}{|l|}{ Result } \\
\hline Shot on target & 10 & 52 & 2 & 16 & 3 & 31 & 3 & 27 & 5 & 41 & $\overline{6}$ & 44 & 5 & $35 \%$ & 3,00 & 10 & $3,34-6,32$ \\
\hline Shot off target & 3 & 14 & 2 & 16 & 2 & 19 & 2 & 14 & 2 & 15 & 3 & 21 & 2 & $16 \%$ & 1,20 & 5 & $1,57-2,76$ \\
\hline Shot blocked & 1 & 5 & 1 & 7 & 1 & 12 & 0 & 3 & 0 & 3 & 1 & 10 & 1 & $6 \%$ & 0,86 & 3 & $0,41-1,26$ \\
\hline Loss of poss. & 5 & 29 & 9 & 64 & 3 & 38 & 7 & 57 & 5 & 36 & 7 & 51 & 6 & $43 \%$ & 2,84 & 9 & $4,64-7,47$ \\
\hline
\end{tabular}

$\mathrm{SD}=$ standard deviation; $\mathrm{CI}=$ confidence interval; $\mathrm{GK}=$ goalkeeper; def. $=$ defender; mid. = midfielder; att. = attacker; $\mathrm{N} / \mathrm{A}=$ not applicable; poss. $=$ possession 
Table 3. Summary of the tactical variables for turf.

\begin{tabular}{|c|c|c|c|c|c|c|c|c|c|c|c|c|c|c|c|c|c|}
\hline \multirow{2}{*}{ Variables } & \multicolumn{2}{|c|}{ U-9 5v5 } & \multicolumn{2}{|c|}{ U-9 7v7 } & \multicolumn{2}{|c|}{ U-9 9v9 } & \multicolumn{2}{|c|}{ U-14 5v5 } & \multicolumn{2}{|c|}{ U-14 7v7 } & \multicolumn{2}{|c|}{ U-14 9v9 } & \multirow{2}{*}{ MEAN } & \multirow{2}{*}{$\%$} & \multirow{2}{*}{ SD } & \multirow{2}{*}{ Range } & \multirow{2}{*}{ CI } \\
\hline & Avg & $\%$ & Avg & $\%$ & Avg & $\%$ & Avg & $\%$ & Avg & $\%$ & Avg & $\%$ & & & & & \\
\hline No. of attacks & 16 & & 13 & & 13 & & 15 & & 17 & & 10 & - & 14 & - & 3,43 & 15 & $12,18-15,59$ \\
\hline \multicolumn{18}{|l|}{ Origin of attack } \\
\hline Defensive half & 10 & 64 & 7 & 56 & 6 & 45 & 11 & 75 & 9 & 56 & 6 & 36 & 8 & $60 \%$ & 3,10 & 11 & $6,74-9,82$ \\
\hline Attacking half & 6 & 36 & 6 & 44 & 7 & 55 & 4 & 25 & 7 & 44 & 4 & 24 & 6 & $40 \%$ & 2,87 & 11 & $4,18-7,04$ \\
\hline \multicolumn{18}{|c|}{ How move was initiated } \\
\hline Counter-attack & 10 & 62 & 9 & 69 & 9 & 70 & 11 & 77 & 12 & 72 & 8 & 48 & 10 & $71 \%$ & 2,54 & 11 & $8,63-11,15$ \\
\hline Static attack & 6 & 38 & 4 & 31 & 4 & 30 & 3 & 23 & 5 & 28 & 2 & 12 & 4 & $29 \%$ & 1,78 & 6 & $3,11-4,89$ \\
\hline \multicolumn{18}{|l|}{ Initiating player } \\
\hline GK & 3 & 21 & 0.3 & 3 & 0.3 & 3 & 3.3 & 23 & 2.3 & 14 & 0.7 & 4 & 2 & $12 \%$ & 1,81 & 6 & $0,82-2,62$ \\
\hline Defender & 6 & 38 & 6 & 44 & 4 & 28 & 6 & 43 & 4 & & 4 & 26 & 5 & $36 \%$ & 2,00 & 8 & $4,01-5,99$ \\
\hline Midfielder & 0 & 0 & 3 & 26 & 8 & 58 & 2 & 14 & 8 & 48 & 4 & 26 & 4 & $30 \%$ & 3,61 & 11 & 2,43-6,02 \\
\hline Attacker & 6 & 40 & 4 & 28 & 2 & 13 & 3 & 20 & 2 & 14 & 1 & 4 & 3 & $21 \%$ & 2,39 & 7 & $1,76-4,13$ \\
\hline \multicolumn{18}{|c|}{ How the ball was pushed forward } \\
\hline Pass & 7 & 45 & 7 & 54 & 8 & 60 & 8 & 52 & 8 & 46 & 8 & 50 & 8 & $55 \%$ & 2,20 & 9 & $6,62-8,71$ \\
\hline Goal kick & 2 & 15 & $\mathbf{0}$ & 0 & $\mathbf{0}$ & 0 & 1 & 5 & 1 & 4 & $\mathbf{0}$ & 0 & 1 & $4 \%$ & 1,24 & 5 & $-0,01-1,23$ \\
\hline GK throw & $\mathbf{0}$ & 2 & $\mathbf{0}$ & 0 & $\mathbf{0}$ & 0 & 1 & 7 & 1 & 4 & $\mathbf{0}$ & 0 & $\mathbf{0}$ & $2 \%$ & 0,84 & 3 & $\begin{array}{ll}-0,08-0,75 \\
\end{array}$ \\
\hline Corner & 2 & 11 & $\mathbf{0}$ & 0 & 2 & 15 & 1 & 9 & 1 & 6 & 1 & 4 & 1 & $8 \%$ & 1,08 & 3 & $0,57-1,65$ \\
\hline Throw-in & 1 & 9 & 3 & 26 & 2 & 13 & 1 & 7 & 2 & 14 & 1 & 6 & 2 & $13 \%$ & 1,31 & 4 & $1,13-2,43$ \\
\hline Free kick & $\mathbf{0}$ & 2 & $\mathbf{0}$ & 3 & $\mathbf{0}$ & 0 & $\mathbf{0}$ & 0 & 0 & 0 & 0 & 0 & $\mathbf{0}$ & $1 \%$ & 0,32 & 1 & $\begin{array}{ll}-0,05-0,27 \\
\end{array}$ \\
\hline Dribble & 2 & 13 & 1 & 10 & 1 & 10 & 2 & 11 & 3 & 18 & 0 & 0 & 2 & $11 \%$ & 1,25 & 3 & $0,94-2,18$ \\
\hline Shot & 0 & 2 & 1 & 8 & 0 & 0 & 0 & 2 & 1 & 6 & 0 & 0 & 0 & $3 \%$ & 0,86 & 3 & $0,02-0,87$ \\
\hline Header & 0 & 2 & 0 & 0 & 0 & 3 & 1 & 5 & 0 & 2 & 0 & 0 & 0 & $2 \%$ & 0,46 & 1 & $0,05-0,51$ \\
\hline Penalty & & 0 & $\mathbf{0}$ & 0 & $\mathbf{0}$ & 0 & $\mathbf{0}$ & 0 & $\mathbf{0}$ & 0 & $\mathbf{0}$ & 0 & $\mathbf{0}$ & $0 \%$ & 0,00 & $\mathbf{0}$ & 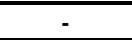 \\
\hline \multicolumn{18}{|c|}{ Area where first pass was received } \\
\hline Defensive half & 5 & 34 & 3 & 26 & 5 & 40 & 6 & 43 & 5 & 32 & 5 & 30 & 5 & $37 \%$ & 2,81 & 11 & $3,72-6,51$ \\
\hline Attacking half & 8 & 49 & 8 & 62 & 7 & 50 & 7 & 48 & 9 & 56 & 4 & 26 & 7 & $52 \%$ & 3,71 & 16 & $5,32-9,01$ \\
\hline N/A & 3 & 17 & 2 & 13 & 1 & 10 & 1 & 9 & 2 & 12 & 1 & 4 & 2 & $12 \%$ & 1,42 & 5 & $0,90-2,32$ \\
\hline \multicolumn{18}{|c|}{ No. of passes in attack } \\
\hline Average & 2 & -1 & 3 & - & 2 & - & 3 & - & 3 & - & 3 & & 3 & - & 0,97 & 3 & $2,18-3,15$ \\
\hline \multicolumn{18}{|c|}{ No. of players involved in attack } \\
\hline Average & 2 & & 3 & & 3 & & 3 & & 3 & & 4 & & 3 & - & 0,54 & 2 & $2,68-3,21$ \\
\hline \multicolumn{18}{|c|}{ Duration of attack } \\
\hline Average & 9 & - & 11 & - & 10 & & 13 & - & 12 & - & 13 & - & 11 & - & 3,27 & 12 & $9,71-12,96$ \\
\hline
\end{tabular}




\begin{tabular}{|c|c|c|c|c|c|c|c|c|c|c|c|c|c|c|c|c|c|}
\hline \multicolumn{18}{|c|}{ Player who ended the attack } \\
\hline Opposition GK & 6 & 40 & 3 & 26 & 4 & 28 & 5 & 34 & 5 & 30 & 2 & 12 & 4 & $30 \%$ & 2,05 & 6 & $3,21-5,24$ \\
\hline Opposition def. & 2 & 13 & 1 & 8 & 4 & 30 & 2 & 14 & 2 & 10 & 4 & 24 & 2 & $18 \%$ & 1,46 & 6 & $1,72-3,17$ \\
\hline Opposition mid. & $\mathbf{0}$ & 0 & $\mathbf{0}$ & 3 & $\mathbf{0}$ & 3 & 1 & 5 & 1 & 8 & $\mathbf{0}$ & 2 & 1 & $4 \%$ & 0,86 & 3 & $0,07-0,93$ \\
\hline Opposition att. & $\mathbf{0}$ & 0 & $\mathbf{0}$ & 0 & $\mathbf{0}$ & 0 & 1 & 5 & $\mathbf{0}$ & 0 & $\mathbf{0}$ & 0 & $\mathbf{0}$ & $1 \%$ & 0,32 & 1 & $-0,05-0,27$ \\
\hline Defender & 1 & 4 & $\mathbf{0}$ & 0 & $\mathbf{0}$ & 3 & 1 & 5 & $\mathbf{0}$ & 0 & $\mathbf{0}$ & 0 & $\mathbf{0}$ & $2 \%$ & 0,57 & 2 & $-0,01-0,56$ \\
\hline Midfielder & $\mathbf{0}$ & 0 & 1 & 8 & 1 & 10 & 1 & 5 & 2 & 14 & 1 & 4 & 1 & $7 \%$ & 1,14 & 4 & $0,43-1,57$ \\
\hline Attacker & 3 & 17 & 3 & 26 & 1 & 10 & 2 & 16 & 2 & 14 & 2 & 12 & 2 & $17 \%$ & 1,46 & 5 & $1,61-3,06$ \\
\hline N/A (Goal) & 4 & 26 & 4 & 31 & 2 & 18 & 3 & 18 & 4 & 24 & 1 & 6 & 3 & $22 \%$ & 1,75 & 6 & $2,13-3,87$ \\
\hline \multicolumn{18}{|l|}{ Result } \\
\hline Shot on target & 7 & 47 & 7 & 51 & 5 & 38 & 5 & 36 & 6 & 36 & 2 & 14 & 5 & $39 \%$ & 2,38 & 9 & $4,26-6,63$ \\
\hline Shot off target & 3 & 19 & 2 & 18 & 2 & 15 & 2 & 16 & 4 & 24 & 1 & 8 & 3 & $18 \%$ & 1,54 & 6 & $1,73-3,27$ \\
\hline Shot blocked & 0 & 0 & 0 & 0 & 1 & 8 & 0 & 2 & 0 & 2 & $\mathbf{0}$ & 0 & $\mathbf{0}$ & $2 \%$ & 0,57 & 2 & $-0,01-0,56$ \\
\hline Loss of poss. & 5 & 34 & 4 & 31 & 5 & 40 & 7 & 45 & 6 & 38 & 6 & 38 & 6 & $41 \%$ & 1,81 & 7 & $4,76-6,57$ \\
\hline
\end{tabular}

$\mathrm{SD}=$ standard deviation; $\mathrm{CI}=$ confidence interval; $\mathrm{GK}=$ goalkeeper; def. $=$ defender; mid. = midfielder; att. = attacker; $\mathrm{N} / \mathrm{A}=$ not applicable; poss. $=$ possession 


\section{Discussion}

There has been a lot of research that has enabled soccer to improve: technique, tactics and strategy through analysis. However to date there have not been any complementary studies objectively analysing the effect of the type of surface on the tactical and strategic elements of soccer. On the data presented all surface types, 5v5 games in the U-9 age group created more attacks than the other age groups/game formats (McNitt et al., 2007). There is a clear tendency for more attacks in 5v5 than in 9v9 games in both age groups on both dirt and artificial pitches. However, on natural grass there were more attacks in a 9v9 format for the U-14 age group. Regarding to the origin of the attacks, the majority began in the defensive half (i.e. the attacking team's own half - on average $58 \%$ ) regardless of the surface type, the age group or the game format. Once again, the $5 \mathrm{v} 5$ format created the greatest variety of moves, with some significant variations on dirt $(73 \%)$ and natural grass $(68 \%)$. Despite $58 \%$ of the attacks originating in the defensive half, the first pass was received in the attacking half on $54 \%$ of occasions. Most of the attacks were initiated with a counter-attack (which in turn originated from a move in continuous play), whereas static attacks (starting from a set piece or a stationary position) were less common (Hughes and Franks, 2005). The majority of attacks were initiated by either a defender or midfielder on all surfaces. The greatest deviation from the average number of passes occurred on natural grass, where the duration of attacks ranged from 8 to 14 seconds. Similarly, tactical analysis suggested that certain variables, for example, frequency of tackles and possession time, were comparable on all surfaces (Ford et al., 2006). It was notable that on both natural grass and artificial turf pitches, the U-14 age group attempted more passes than the U-9 age group (on natural grass an average of 83 compared to 65, and on artificial turf an average of 88 compared to 59). Furthermore, within these values, the percentages of passes that successfully reached a team-mate was much higher in the U-14 age group than in the U-9 (on natural grass an average of $87 \%$ compared to $64 \%$, and on artificial turf an average of $83 \%$ compared to $73 \%$ ). In the U-9 age group, the ball was out of play for less time on the dirt pitches and for more time on the artificial turf. In the U-14 age group, however, the ball was out of play for less time on the artificial turf and for more time on the dirt pitches. This variation can be explained by the fact that the players in the U-14 age group had a higher level of technical skill than the U-9 players (Lees, 1996). It should also be taken into acount that the data collected from the U-14 age group showed higher values for surfaces that facilitate passing and building attacking play (artificial and natural turf).

Having discussed the most representative values for the tactical and strategic moves reflected in the different variables of this study, it is concluded that all of them are closely interrelated and change uniformly depending principally on the type of playing surface, but also on the game format and age group (Dixon et al., 1999). As stated above, all game formats and age groups follow a logical evolution, developing from reduced to bigger sizes and therefore having a direct impact on the tactical performances. Therefore, they cannot be seen as isolated actions but rather as part of a common trend (Di Lorenzo et al., 1998). One of the most important objectives for all coaches is the high-level technical and tactical development of their players, because in most cases, it is not the competitors with the most stamina, strength, speed or joint mobility who win, and neither is it the players with the best technical delivery from a 
biomechanical point of view, but rather it is the athlete who is able to grasp the different and varied situations that occur during a match, analyse them correctly and make the appropriate technical-tactical move, assessing his own situation in relation to his opponent and those around him (Sallis et al., 1997). It is fair to say that all the players (from both teams) had a direct influence on the games' final results, depending on their behaviour and their decision-making from a tactical point of view. It has been speculated that differences in performance on the pitch are not only due to the type of surface, but also to factors such as age of the pitch and construction methods (Alcántara et al., 2009). In addition, many of the differences among grass, dirt and artificial turf could be attributed not only to the surface but also to the players' distinct styles (Naunheim et al, 2004).

\section{Conclusions}

Artificial turf pitches generate more attacks. Greater levels of attacking play are generated on artificial turf pitches - in fact, the artificial turf pitches generated higher levels of positive data in almost all the variables (Dumlop, 2001). On average, players had more touches of the ball on artificial turf than on natural grass or dirt pitches. Moreover, according to the answers given by the players on the forms, they themselves also had the impression that they had had more touches of the ball on artificial turf. $35 \%$ of respondents estimated that they had had more than 50 touches on artificial turf, compared to $19 \%$ on natural grass and $13 \%$ on the dirt pitch. On all surfaces, a clear trend emerged, which was that there were more attacks (goals, shots on goal, one-onones and entries into the penalty area) in the smaller-sided game formats (5v5 and $7 v 7$ ) than in $9 \mathrm{v} 9$ games. It should be particularly noted that in the U-9 age group playing $5 \mathrm{v} 5$, more attacks and attacking moves were created than in any other age group and game format, on all surfaces. Despite the average number of touches by each player on the pitch being lower on artificial turf, a greater average number of attempted attacks were seen on this surface than on any other; the average number of attacks was greater on artificial turf than on dirt or natural grass. Moreover, the number of touches per outfield player was considerably higher in the $5 \mathrm{v} 5$ game format than in the $9 \mathrm{v} 9$ format across all surfaces and both age groups. This trend was also observed in the case of goalkeepers whose average number of touches of the ball in the $5 \mathrm{v} 5$ format was clearly greater than in the $9 \mathrm{v} 9$ format, on all surfaces.

The players preferred playing on natural grass or artificial turf pitches to dirt pitches: the feedback received from the players immediately after playing revealed a marked preference for natural grass or artificial pitches over dirt pitches (Foster, 2007). 96\% of the players enjoyed playing on natural grass or artificial turf, in comparison to $52 \%$ who said they enjoyed playing on dirt; $80 \%$ of the players did not think that dirt was a good surface to play on, whereas $100 \%$ said that play was more fluid on artificial turf and 95\% thought the same for natural grass. Specific feedback suggests that the reason the players did not enjoy playing on a dirt pitch was the uneven surface which made it difficult to play on, slippery and meant it had an uneven bounce. Conversely, the players found the natural grass and artificial turf pitches optimal to play on thanks to the even surface and the fact that it did not hurt when they fell over (Catón, 2004). 


\subsection{Practical implications}

The practical application of the results presented is especially complex due to the high number of variables that influence just one individual or collective tactical move, and due to the effect of the surface and the game format on the intensity in terms of said variables (Kelly and Drust, 2009). Although practical applications for the tactical behaviour of players on different surfaces have been proposed, most studies into the use of artificial surfaces have focused upon the incidence and severity of injuries (Fuller et al, 2007a). On the other hand, there are biomechanical studies, both theoretical and experimental (Fuller et al, 2007b; Dimichele et al, 2009; Aoki et al, 2010) that may be useful in determining the interaction of the players with the different playing surfaces (Nigg \& Yeadon, 1987; Ekstrand \& Nigg, 1989; Walden et al, 2005; Ekstrand et al, 2006) and the possible tactical repercussions. The predominant perception among the players who participated in the study was that artificial turf stood out as a surface that was superior to standard natural grass and to dirt. However, there are studies that have indicated a relationship among the players' preferences for types of surface and the weather conditions (Zanetti, 2009). In general, players prefer to play on artificial surfaces when temperatures are very high or very low, despite the risk of injury in high temperatures and the greater risk of abrasion (Willwock et al, 2009), but in rain they prefer natural surfaces. On the other hand, depending on the type of playing style a team wishes to develop, certain surfaces will be more conducive than others both for training and for competitions (Meyers and Barnhill, 2004). For example, natural grass and artificial turf favour an attacking game based on ball possession, using attacking tactics and combined attacks (with mainly short and low passes), whereas dirt pitches favour play with short possession and more direct attacks, applying attacking tactics by getting past tactical formations using mainly long and aerial passes.

It is hoped that the objective analysis provided by this study will provide relevant information for interested parties working in youth soccer development. The authors declare that they have no conflicts of interests related to the contents of the study. The results of this study do not constitute endorsement of the products and equipment used by the authors.

\section{Acknowledgements}

The material submitted is original and unpublished, and is not under consideration for publication elsewhere; the material will not be submitted for publication elsewhere while it is under consideration by the journal.. The Polytechnic University of Madrid's Institutional Review Board approved the use of human subjects in this research for the purpose of collecting all of the data and statistically analysing it for the study. The study was endorsed by the Fédération Internationale de Football Association (FIFA) and has also been supported by the Royal Spanish Football Federation Association (RFEF). In addition, FIFA has donated sports equipment (balls, bibs, cones, markers and minigoals) to the clubs involved in the study. The authors declare that they have no conflicts of interests related to the contents of the study. 


\section{References}

Abrantes, C.I., Nunes, M.I., Maçãs, V.M., Leite, N.M., \& Sampaio, J.E. (2012). Effects of the number of players and game type constraints on heart rate, rating of perceived exertion, and technical actions of small-sided soccer games. Journal of Strength and Conditioning Research, 4, 976-981.

Alcántara, E., Gámez, J., Rosa, D., \& Sanchís, M. (2009). Analysis of the influence of rubber infill morphology on the mechanical performance of artificial turf surfaces for soccer. Proceedings of the Institution of Mechanical Engineers, Part P: Journal of Sports Engineering and Technology, 223, 1-9.

Andersson, H., Ekblom, B., \& Krustrup, P. (2008). Elite football on artificial turf versus natural grass: movement patterns, technical standards, and player impressions. Journal of Sports Sciences, 26, 113-122.

Aoki, H., Kohno, T., Fujiya, H., Kato, H., Yatabe, K., Morikawa, T., \& Seki, J. (2010). Incidence of injury among adolescent soccer players: A comparative study of artificial and natural grass turfs. Clinic Journal of Sports Medicine, 20, 1-7.

Atkinson, G. (2001). Sport performance: variable or construct? Journal of Sports Sciences, 20, 291-292.

Casamichana, D., \& Castellano, J. (2010). Time-motion, heart rate, perceptual and motor behaviour demands in small-sided soccer games: Effects of pitch size. Journal of Sports Sciences, 28, 1615-1623.

Catón, J. (2004). Consolidation of artificial turf. Instalaciones Deportivas XXI, 131, 26-29.

Choi, H., O’Donoghue, P., \& Hughes, M. (2007). An investigation of inter-operator reliability tests for real-time analysis system. International Journal of Performance Analysis in Sport, 7, 49-61.

Claudio, L. (2008). Synthetic turf health debate takes root. Environmental Health Perspectives, 116, 116-122.

DiMichele, R., DiRenzo, A. M., Ammazzalorso, S., \& Merni, F. (2009). Comparison of the physiological responses to an incremental running test on treadmill, natural grass, and synthetic turf in young soccer players. Journal of Strength and Conditioning Research, 23, 939-945.

Di Lorenzo, T. M., Stucky-Ropp, R. C., Vander Wal, S.J., \& Gotham, H. J. (1998). Determinants of exercise among children. II. A longitudinal analysis. Preventive Medicine, 27, 470-477.

Dixon, S. J., Batt, M. E., \& Collop, A. C. (1999). Artificial playing surfaces research: a review of medical, engineering and biomechanical aspects. International Journal of Sports Medicine, 20, 209-218.

Dumlop, J. (2001). Wear and care: assessing the benefits of synthetic surfaces. Stadia, $8,74-76$.

Duncan, S., Duncan, T., Stryker, L., \& Chaumeton, N. (2002). Neighbourhood physical activity opportunity: a multilevel contextual model. Research Quarterly for Exercise and Sport, 73, 457-463.

Ekstrand, J., \& Nigg, B. M. (1989). Surface-related injuries in soccer. Sports Medicine, $8,56-62$.

Ekstrand, J., Timpka, T., \& Hagglund, M. (2006). Risk of injury in elite football played on artificial turf versus natural grass: a prospective two-cohort study. British Journal of Sports Medicine,40, 975-980. 
Ekstrand, J. (2008). Epidemiology of football injuries. Science and Sports, 23, 73-77.

Fédération Internationale de Football Association (2010). Manager's guide to Natural Grass Football Pitches. Zurich: FIFA Member Associations and Development, 12-14.

Fédération Internationale de Football Association (2012). Grassroots manual. Zurich: FIFA Education and Technical Development, 65-69.

Fédération Internationale de Football Association (2013). FIFA quality concept for football turf. Zurich: FIFA Member Associations and Development, 41-45.

Ford, K., Manson, N., Evans, B., Myer, G., Gwin, R.., \& Heidt, R. (2006). Comparison of in-shoe foot loading patterns on natural grass and synthetic turf. Journal of Science and Medicine in Sport, 9, 433-440.

Foster, J. B. (2007). Newer artificial turf appears safer for soccer players. Biomechanics, 14, 9-10.

Fuller, C. W., Dick, R. W., Corlette, J., \& Schmalz, R. (2007a). Comparison of the incidence, nature and cause of injuries sustained on grass and new generation artificial turf by male and female football players. Part 1: match injuries. British Journal of Sports Medicine, 41, 20-26.

Fuller, C. W., Dick, R. W., Corlette, J., \& Schmalz, R. (2007b). Comparison of the incidence, nature and cause of injuries sustained on grass and new generation artificial turf by male and female football players. Part 2: training injuries. British Journal of Sports Medicine, 41, 27-32.

Hadley, L., Poitras, M., Ruggiero, J., \& Knowles, S. (2000). Performance evaluation of national football league teams. Managerial and Decision Economics, 21, 4556.

Hill-Haas, S. V., Rowsell, G. J., Dawson, B. T., \& Coutts, A. J. (2009). Acute physiological responses and time-motion characteristics of two small-sided training regimes in youth soccer players. Journal of Strength and Conditioning Research, 23, 111-115.

Hill-Haas, S. V., Coutts, A. J., Dawson, B. T., \& Rowsell, G. J. (2010). Time-motion characteristics and physiological responses of small-sided games in elite youth players: The influence of player number and rule changes. Journal of Strength and Conditioning Research, 24, 2149-2156.

Hughes, M., \& Bartlett, R. (2002). The use of performance indicators in performance analysis. Journal of Sports Sciences, 20, 739-754.

Hughes, M., \& Franks, I. (2005). Analysis of passing sequences, shots and goals in soccer. Journal of Sports Sciences, 23, 509-514.

James, N., Taylor, J., \& Stanley, S. (2007). Reliability procedures for categorical data in Performance Analysis. International Journal of Performance Analysis in Sport, 7, 1-11.

Kelly, D. M., \& Drust, B. (2009). The effect of pitch dimensions on heart rate responses and technical demands of small-sided soccer games in elite players. Journal of Science and Medicine in Sport, 12, 475-479.

Lees, A. (1996). Biomechanics applied to soccer skills. Science and Soccer. London: E \& FN Spon, 123-134.

Lees, A., \& Nolan, L. (1998). The biomechanics of soccer: a review. Journal of Sports Sciences, 16, 211-234.

McNitt, A. S., Petrunka, D., \& Hardness, S. (2007). Evaluation of playing surface characteristics of various in-filled systems. Evaluation, 9, 20-32. 
Meyers, M., \& Barnhill, B. (2004). Incidence, causes, and severity of high school football injuries on FieldTurf vs. natural grass: A 5-year prospective study. American Journal of Sports Medicine, 32, 1626-1638.

Naunheim, R., Parrott, H., \& Standeven, J. (2004). A comparison of Artificial Turf. The Journal of Trauma Injury Infection and Critical Care, 57, 1311-1314.

Nigg, B. M., \& Yeadon, M. R. (1987). Biomechanical aspects of playing surfaces. Journal of Sports Sciences, 5, 117-145.

O'Donoghue, P. (2005). Normative profiles of sports performance. International Journal of Performance Analysis in Sport, 5, 104-119.

O'Donoghue, P., Bradley, P., Tordoff, P., \& Wooster, B. (2007). The reliability of ProZone MatchViewer: A video-based technical performance analysis system. International Journal of Performance Analysis in Sport, 7, 117-129.

O'Donoghue, P. (2009). Interacting performances theory. International Journal of Performance Analysis in Sport, 9, 26-46.

Orchard, J. (2002). Is there a relationship between ground and climatic conditions and injuries in football? Sports Medicine, 32, 419-432.

Platt, D., Maxwell, A., Horn, R., Williams, M., \& Reilly, T. (2001). Physiological and technical analysis of $3 \vee 3$ and 5 v 5 youth football matches. Insight: The FA Coaches Association Journal, 4, 23-24.

Rampinini, E., Impellizzeri, F. M., Castagna, C., Abt, G., Chamari, K., Sassi, A., \& Marcora, S. M. (2007). Factors influencing physiological responses to smallsided soccer games. Journal of Sports Sciences, 25, 659-666.

Reilly, T., Williams, A. M., Nevill, A., \& Franks, A. (2000). A multidisciplinary approach to talent identification in soccer. Journal of Sports Sciences, 18, 695702.

Sallis, J. F., Johnson, M. F., Calfas, K. J., Caparosa, S., \& Nicols, J. F. (1997). Assessing perceived physical environmental variables that may influence physical activity. Research Quarterly for Exercise and Sport, 68, 345-351.

Sanchís, M., Rosa, D., Magraner, L., Ortega, N., \& Muñoz, B. (2008). MULTITURF: Innovation in multisport artificial turf pitches. Revista de Biomecánica, 49, 911.

Schmidt-Oltsen, S., Jörgensen, U., Kaalund, S., \& Sörensen, J. (1991). Injuries among young soccer players. American Journal of Sports Medicine, 19: 273-275.

Steffen, K., Andersen, T. E., \& Bahr, R. (2007). Risk of injury on artificial turf and natural grass in young female football players. British Journal of Sports Medicine, 1, 1-6.

Stiles, V. H., James, I. T., Dixon, S. J., \& Guisaola, I. N. (2009). Natural turf surfaces: the case for continued research. Sports Medicine, 39: 65-84.

Tessitore, A., Meeusen, R., Piacentini, M. F., Demarie, S., \& Capranica, L. (2006). Physiological and technical aspects of " 6 -a-side" soccer drills. Journal of Sports Medicine and Physical Fitness, 46, 36-43.

Walden, M., Hagglund, M., \& Ekstrand, J. (2001). UEFA Champions League study: a prospective study of injuries in professional football during the 2001-2002 season. British Journal of Sports Medicine, 39: 542-546.

Willwock, M. R., Meyer, E. G., Powell, J. W., Fouty, A. J., \& Haut, R. C. (2009). Football playing surface and shoe design affect rotational traction. American Journal of Sports Medicine, 37, 518-525. 
Zanetti, E. M. (2009). Amateur football game on artificial turf: Players' perceptions. Applied Ergonomics, 40, 485-490.

\section{Corresponding author:}

Jorge Diaz-Cidoncha Garcia

Affiliation: Fédération Internationale de Football Association (FIFA). Education and Technical Development Department. / National Institute of Physical Education (INEF), Polytechnic University of Madrid. Sports Department.

Mailing Address: Niederdorfstrasse 48. 8001 Zurich. Switzerland.

Telephone number: +41 (0) 793689252 / +41 (0) 432227777

Fax: +41-(0)43-222 7878

Email address: jorge.diaz@ fifa.org 